\title{
Cardiovascular Risk Among University Students from Developed and Developing Nations
}

\author{
Barry E. Bleske ${ }^{1, *}$, Steven R. Erickson ${ }^{1}$, Sahar Fahoum ${ }^{2}$, Krishna R. Devarakonda ${ }^{3}$, Lynda S. Welage ${ }^{1}$, \\ Marah Koudmani ${ }^{2}$, Narayan Pantham ${ }^{4}$, Stephanie B. Edwin ${ }^{5}$, Siddhartha Devarakonda ${ }^{6}$, \\ Michael J. Shea ${ }^{7}$, Srinivas Martha ${ }^{8}$ and Nabil Khalidi ${ }^{1}$ \\ ${ }^{I}$ Department of Clinical and Social Administrative Sciences, College of Pharmacy, University of Michigan, USA \\ ${ }^{2}$ College of Pharmacy, University of Kalamoon, Deratiah, Syria \\ ${ }^{3}$ Covidien, Hazelwood, Missouri, USA \\ ${ }^{4}$ Department of Medicine, Kakatiya Medical College, Warangal, AP, India \\ ${ }^{5}$ Department of Pharmacy, St. John Hospital, USA \\ ${ }^{6}$ Gandhi Medical College, Hyderabad, AP, India \\ ${ }^{7}$ Department of Internal Medicine, Division of Cardiology, University of Michigan Health System, USA \\ ${ }^{8}$ University College of Pharmaceutical Sciences, Kakatiya University, Warangal, AP, India
}

\begin{abstract}
Background: A key aspect in halting global increase in cardiovascular events is prevention and especially prevention at an early age. Unfortunately, global data regarding cardiovascular risk factors in the young are limited. Therefore the objectives of this study were to identify the most common cardiovascular risk factors among young adults in a university setting in both developed and developing countries.

Methods: Lifestyle and cardiovascular risk factors (smoking status, rates of physical activity, alcohol use, family history, blood pressure, fasting lipid panel, fasting blood glucose) were prospectively evaluated in young adults at three different university settings [University of Michigan (Ann Arbor, USA), University of Kalamoon (Deratiah, Syria), and Kakatiya University (Warangal, India)].

Results: A total of 296 subjects (mean age and standard deviation $22 \pm 3$ years) were evaluated. Rates of current smoking were markedly higher $(\mathrm{p}<0.001)$ in Syria $(43 \%)$ compared with the USA $(6.2 \%)$ and India $(1.7 \%)$. Subjects in India were significantly $(\mathrm{p}<0.001)$ less likely to engage in physical activity $(20.2 \%)$ compared with the USA $(90.7 \%)$ and Syria $(68.8 \%)$. Fasting blood glucose levels and body mass index were significantly higher $(\mathrm{p}<0.001)$ in Syria as compared to other countries. Significant differences were also noted in LDL, HDL, and triglycerides among the three sites.

Conclusions: Cardiovascular risk factors among young adults in a university setting vary depending on global setting. Based upon the results of this study, targeted interventional programs based on risk findings from individual countries may be a reasonable future strategy to help reduce long term cardiovascular morbidity and mortality.
\end{abstract}

Keywords: Lipids, Young, Global, Glucose, BMI.

\section{INTRODUCTION}

The incidence of morbidity and mortality associated with cardiovascular disease (CVD) is significant not only for developed countries but also in developing countries [1,2]. According to the latest World Health Organization (WHO) statistics CVD is responsible for a third of global deaths [1]. Low and middle income countries bear over $82 \%$ of the deaths associated with CVD. CVD is the leading cause of death worldwide. The social and economic impact of CVD

*Address correspondence to this author at the University of Michigan, College of Pharmacy 428 Church Street Ann Arbor, Michigan, 48109-1065;

Tel: (734) 764-5341; Fax: (734) 763-4480; E-mail: bbleske@umich.edu is apparent in developed nations; however, it is more acutely felt in developing nations [1]. CVD tend to affect individuals in their peak earning and productive years. The economic consequence associated with CVD is not only devastating to the family but also to the country since the early loss of human resources can dramatically affect intellectual and economic output $[1,2]$. In addition, the cost of tertiary medical therapy must also be considered. These findings clearly show the need to identify the most important risk factors associated with CVD in a global fashion and to develop cost effective interventional programs.

Even though CVD is approaching epidemic proportions there is tremendous disparity in conducting research and 
intervention on a global scale especially in developing nations. In fact, when looking at the global burden of disability adjusted life years (DALY), a $55 \%$ increase in DALY loss attributable to CVD between 1990 and 2020 is predicted by the Global Burden of Disease Study for lowincome and low-middle-income countries (i.e. developing nations) [2-4]. In contrast, developed nations or high-income nations will likely see a $14.3 \%$ reduction. This reduction is likely due to research and interventions conducted in these countries. In a paper by Prabhakaran, et al. addressing this issue suggests that effective national policies need to be put in place for the prevention of CVD [5]. In addition, it was suggested that for national policies to be established, research needs to be done in country. One of the important aspects of our study follows this recommendation in that to better understand the CVD risk in a global perspective research was performed by investigators living and working in each of the countries studied. This type of approach will hopefully lead to better understanding and greater insight in developing nation specific interventional programs to prevent CVD.

Another important aspect of this study is to not only conduct research in country but to identify the cardiovascular risk factors associated with a country's young population. As with any type of disease the best defense is prevention. Awareness and appropriate life style choices regarding CVD can have significant impact in regard to the development of CVD, especially the development of early disease. The primary goals of this study are to determine and compare cardiovascular risk factors in different countries among the young. Specifically for this initial study, we primarily evaluated the young and educated (i.e. university students). Understanding the risk factors associated with a younger population may allow the ability to develop a targeted and cost effective interventional program that will potentially have the greatest impact on CVD since emphasis will be placed on primary prevention at a time when it is most advantageous. Another important aspect of this study is that we are evaluating a population where risk is not well known and certainly not well known on a global level.

\section{METHODS}

To assess cardiovascular risk factors among young adults, subjects were recruited from the University of Michigan (Ann Arbor, USA), the University of Kalamoon (Deratiah, Syria), and Kakatiya University (Warangal, India). Subjects between 18 and 35 years old who were residents and lived in the site country for at least one year were screened for inclusion. Subjects who regularly took prescription or nonprescription medications, had significant past medical history, or were pregnant were excluded. Subjects were recruited by advertisements and were taken in a consecutive fashion.

This study protocol was approved by the appropriate regulatory and ethics committees in all participating countries. All subjects were provided an informed consent document prior to participation in the study. Each subject was required to fast for a minimum of 8 hours prior to the cardiovascular assessment.

Structured questionnaires and physical assessments were systematically conducted for each subject. Standardized training materials (written and video) along with identical equipment were used at all three sites. Information pertaining to demographics, education (university student, health care student), lifestyle (smoking status, physical activity, and alcohol use), ethnicity, and family history of cardiovascular disease was collected from each subject. A minimum of three blood pressure readings were collected for each subject with an automated blood pressure machine (Omron HEM-780, Omron Healthcare Inc., Bannockburn, IL). For subjects with an arm circumference less than $27 \mathrm{~cm}$ or greater than $44 \mathrm{~cm}$, a manual blood pressure monitor was utilized. To assure consistency, diastolic blood pressure needed to be within $10 \mathrm{mmHg}$ for each of the three readings. The average of the three blood pressures was then determined and reported. A fasting lipid panel was obtained for each subject with a point-of-care testing device (Cardiochek PA, Polymer Technology Systems Inc., Indianapolis, IN), along with a fasting blood glucose level (OneTouch Ultra, Milpitas, CA). Height and waist circumference were measured to the nearest $0.1 \mathrm{~cm}$ with a non-stretchable measuring tape (Gulick, Lafayette Instruments, Lafayette, IN). Subject weight was measured to the nearest $0.1 \mathrm{~kg}$ with a digital scale (Omron HBF-500, Omron Healthcare Inc., Bannockburn, IL). Height, weight, and waist circumference were measured while subjects were wearing clothing, but no shoes. Body mass index was calculated for each subject in the study.

In this study, family history of cardiovascular disease was defined as definite myocardial infarction or sudden death before 55 years old in father or other first degree male relative (or before 65 years old in mother or other first degree female relative). Current smoker was defined as an individual who had smoked any tobacco in the previous 12 months (including those who had quit smoking within the last year). Former smoker was defined as a previous smoker who had quit smoking for more than a year. Regular alcohol use was defined as consumption of alcohol 3 or more times per week. Intensity of physical activity was measured by three questions [6]. Vigorous physical activity (i.e. running, lap swimming, aerobics classes, fast bicycling) was defined as at least 10 minutes of activity which caused heavy sweating, large increases in breathing, or large increases in heart rate over the past 30 days. Moderate physical activity (i.e. brisk walking, golfing, dancing) was defined as at least 10 minutes of activity which caused only light sweating, a slight to moderate increase in breathing, or a slight to moderate increase in heart rate. Strength activity (i.e. lifting weights, push-ups, sit-ups) was defined as activities specifically defined to strengthen muscles.

Descriptive statistics included mean with standard deviation for continuous variables and frequency with percent for categorical variables. One-way ANOVA was used to determine whether the differences between the mean values of continuous variables based on country were significantly different. The Scheffe post-hoc analysis was performed to examine pairwise differences among the three groups. Chisquare tests were performed to determine whether the overall differences in frequencies of categorical variables were different among the three groups and also for pair wise comparison of differences in frequencies. One of the limitations of the Cardiochek PA device used to test for lipid profiles is 
that LDL is calculated and that triglyceride levels below 50 $\mathrm{mg} / \mathrm{dL}$ are not reported. Therefore, in subjects with triglycerides below $50 \mathrm{mg} / \mathrm{dL}$ neither a triglyceride value nor a LDL value was reported out. As noted in the result section below a number of subjects in each country fit this profile. In these cases, a triglyceride value of $49 \mathrm{mg} / \mathrm{dL}$ was assigned and an LDL value was subsequently calculated from a standard formula [7].

\section{RESULTS}

Among the three countries, 296 subjects were deemed enrolled in the study. Baseline demographic criteria of enrolled subjects are reported in Table 1 . The average age of the subjects was $23 \pm 3$ years old. The majority of subjects enrolled in the study were female $(59 \%)$. The majority of subjects were born in the respective site country in every group. Of note, $30 \%$ of the subjects from the United States were born elsewhere compared to $5 \%$ of subjects from Syria and no subjects from India. Lifestyle and family history of subjects are represented in Table 2. Syria had a much higher incidence of current smokers (43\%) compared to subjects from the United States (6.2\%) and India (1.7\%). Subjects from India engaged in vigorous, moderate, and strength-training exercise less frequently than subjects in the
United States or Syria. Alcohol use was most prevalent in the United States. Subjects with a family history of cardiovascular disease were much more prevalent in Syria $(67.5 \%)$ compared with subjects in the United States (7.2\%) or India (19.3\%).

Blood pressure readings collected throughout the study are reported in Table 3. No significant differences were noted between the three countries pertaining to systolic blood pressure $(\mathrm{p}=0.453)$ or diastolic blood pressure $(\mathrm{p}=0.144)$. When placed in the context of the Seventh Report of the Joint National Committee on Prevention, Detection, Evaluation, and Treatment of High Blood Pressure (JNC7) guidelines, there was a surprisingly high incidence of pre-hypertension (systolic blood pressure $120-139 \mathrm{mmHg}$ or diastolic blood pressure $80-90 \mathrm{mmHg}$ ) or hypertension (systolic blood pressure $\geq 140 \mathrm{mmHg}$ or diastolic blood pressure $\geq 90 \mathrm{mmHg}$ ) across this young adult population (31\% United States, 34\% Syria, 22\% India, $p=0.138$ ) [8].

The results of cholesterol measurement are represented in Table 3. No significant differences were seen in total cholesterol levels among the three countries. Significant differences were noted between the three countries in LDL $(\mathrm{p}<0.001)$, HDL $(\mathrm{p}<0.020)$, and triglycerides $(\mathrm{p}<0.001)$

Table 1. Demographic Characteristics (Data Reported as Mean and Standard Deviation)

\begin{tabular}{|c|c|c|c|c|}
\hline & United States (n=97) & Syria $(n=80)$ & India $(n=119)$ & Total $(n=296)$ \\
\hline Age (years) & $21.92 \pm 3.25$ & $22.88 \pm 3.10$ & $23.11 \pm 3.71$ & $22.66 \pm 3.44$ \\
\hline Male & $33(34 \%)$ & $40(50 \%)$ & $48(39.7 \%)$ & $121(41 \%)$ \\
\hline Born in Site Country & $68(70.1 \%)$ & $76(95 \%)$ & $119(100 \%)$ & $263(89 \%)$ \\
\hline African American & $6(6.2 \%)$ & $0(0 \%)$ & $0(0 \%)$ & $6(2 \%)$ \\
\hline Asian & $22(22.7 \%)$ & $0(0 \%)$ & $0(0 \%)$ & $22(7 \%)$ \\
\hline Caucasian/White & $61(62.9 \%)$ & $0(0 \%)$ & $0(0 \%)$ & $61(21 \%)$ \\
\hline Eastern Indian & $4(4.1 \%)$ & $0(0 \%)$ & $119(100 \%)$ & $123(42 \%)$ \\
\hline Latino/Hispanic & $2(2.1 \%)$ & $0(0 \%)$ & $0(0 \%)$ & $2(0.7 \%)$ \\
\hline Middle Eastern & $2(2.1 \%)$ & $80(100 \%)$ & $0(0 \%)$ & $82(28 \%)$ \\
\hline
\end{tabular}

Table 2. Lifestyle and Family History

\begin{tabular}{|l|l|l|l|l|}
\hline & United States & Syria & India & P value \\
\hline \hline Current Smoker & $6(6.2 \%)$ & $34(43 \%)^{1}$ & $2(1.7 \%)$ & $<0.001$ \\
\hline Former Smoker & $8(8.2 \%)$ & not reported & $2(1.7 \%)$ & 0.024 \\
\hline Vigorous Exercise & $77(79.4 \%)$ & $50(62.5 \%)$ & $29(24.4 \%)^{2}$ & $<0.001$ \\
\hline Moderate Exercise & $88(90.7 \%)$ & $55(68.8 \%)$ & $24(20.2 \%)^{2}$ & $<0.001$ \\
\hline Strengthening Exercise & $67(69.1 \%)$ & $42(52.5 \%)$ & $8(6.7 \%)^{2}$ & $<0.001$ \\
\hline Alcohol Use & $9(9.0 \%)^{3}$ & $0(0 \%)$ & $4(3.4 \%)$ & $<0.001$ \\
\hline Family History of CVD & $7(7.2 \%)$ & $54(67.5 \%)^{1}$ & $23(19.3 \%)^{4}$ & $<0.001$ \\
\hline
\end{tabular}

$\mathrm{CVD}=$ cardiovascular disease $;=\mathrm{p}<0.001$ compared to United States and India $; 2=\mathrm{p}<0.03$ compared to United States and Syria; $3=\mathrm{p}=0.005$ compared to Syria; $4=\mathrm{p}=0.01$ compared to United States. 
Table 3. Cardiovascular Risk Profile (Data Reported as Mean and Standard Deviation)

\begin{tabular}{|l|l|l|l|l|}
\hline & United States & Syria & India & p-value \\
\hline \hline Systolic BP (mmHg) & $109.7 \pm 11.6$ & $111.9+15.1$ & $110.1 \pm 10.6$ & 0.453 \\
\hline Diastolic BP (mmHg) & $73.1 \pm 8.3$ & $74.5 \pm 10.2$ & $72.0 \pm 7.6$ & 0.144 \\
\hline Pre-HTN & $26(26.8 \%)$ & $21(26.2 \%)$ & $23(19.3 \%)$ & 0.25 \\
\hline HTN & $4(4.1 \%)$ & $6(7.5 \%)$ & $3(2.5 \%)$ & 0.2 \\
\hline Pre-HTN or HTN & $30(30.9 \%)$ & $27(33.8 \%)$ & $26(21.8 \%)$ & 0.138 \\
\hline Total Cholesterol (mg/dL) & $156 \pm 31$ & $153 \pm 28$ & $162 \pm 32$ & 0.14 \\
\hline LDL (mg/dL) & $97 \pm 24$ & $99 \pm 26$ & $113 \pm 29^{1}$ & $<0.001$ \\
\hline HDL (mg/dL & $41 \pm 13$ & $40 \pm 19$ & $35 \pm 14^{2}$ & 0.02 \\
\hline Triglycerides (mg/dL) & $88 \pm 67$ & $75 \pm 50$ & $67 \pm 31^{3}$ & $79 \pm 10$ \\
\hline Blood Glucose (mg/dL) & $86 \pm 9$ & $96 \pm 11^{4}$ & $21.5 \pm 2.7$ & $<01$ \\
\hline BMI (kg/m $\left.{ }^{2}\right)$ & $22.9 \pm 3.2$ & $25.2 \pm 4.7^{4}$ & $<0.001$ \\
\hline
\end{tabular}

$1=\mathrm{p}<0.001$ compared to Syria and United States $; 2=\mathrm{p}=0.034$ compared to United States $; 3=\mathrm{p}=0.01$ compared to United States $; 4=\mathrm{p}<0.001$ compared to United States and India Pre-HTN = pre-hypertension, defined as systolic blood pressure $120-139 \mathrm{mmHg}$ or diastolic blood pressure $80-90 \mathrm{mmHg}$.

HTN $=$ hypertension, defined as systolic blood pressure $\geq 140 \mathrm{mmHg}$ or diastolic blood pressure $\geq 90 \mathrm{mmHg}$

Data reported as mean and standard deviation.

levels. The mean LDL reported for India $(113 \pm 28 \mathrm{mg} / \mathrm{dL})$ was markedly higher than mean values from the United States $(97 \pm 24 \mathrm{mg} / \mathrm{dL})$ and Syria $(99 \pm 26 \mathrm{mg} / \mathrm{dL})$. In addition, India has significantly lower HDL as compared to the United States. In regard to triglycerides, the subjects from the United States had the highest levels and those from India the lowest. India also had the highest number of subjects (43\%) reporting triglyceride levels below $50 \mathrm{mg} / \mathrm{dL}$ as compared to Syria (30\%) and the United States (32\%, $\mathrm{p}>0.05)$.

Mean fasting blood glucose levels $(p<0.001)$ were significantly different between the countries with Syria having the highest levels (Table 3). The mean blood glucose level in Syria $(96 \pm 11 \mathrm{mg} / \mathrm{dL})$ was especially concerning, approaching the American Diabetes Association (ADA) definition of impaired fasting glucose (100-125 mg/dL) [9]. Notably, the incidence of impaired fasting glucose per the ADA definition was $39 \%$ of subjects in Syria, compared with 5\% of subjects in the United States and 3\% of subjects in India ( $p<0.001$, Syria versus United States and India). This data corresponds well with body mass index data; Syria, the country with the highest mean blood glucose, also had the highest mean body mass index of the three countries at $25 \pm 5 \mathrm{~kg} / \mathrm{m}^{2}$ (Table 3, Fig. 1). According to the World Health Organization, the mean BMI for Syria is classified as overweight [10].

\section{DISCUSSION}

Significant differences in LDL, HDL, triglycerides, fasting blood glucose, lifestyle factors and body mass index were shown between young adult subjects in a University setting residing in the United States, Syria, and India. Rates of current smoking were noted to be markedly higher in Syria, which may represent a cultural difference between the three countries. Subjects in India were less likely to engage in moderate physical activity, vigorous physical activity, or strengthening activity compared to subjects from the other countries. The reduced physical activity may be associated with potential cardiovascular consequences in this study, as reduced HDL was also noted in this population. The incidence of pre-hypertension or hypertension in the overall study population is concerning, possibly lending itself to a future cardiovascular risk intervention. Significant differences pertaining to body mass index and blood glucose were also noted between the countries. More interesting, however, was the elevated mean fasting glucose noted in Syria. The elevated mean fasting blood glucose documented in the Syrian subjects was associated with the high mean body mass index calculated for this cohort. These data supports the increasing rates of metabolic syndrome in developing nations noted in previous studies [11,12].

The data collected in this study represents cardiovascular risk factors that translate globally as demonstrated by the

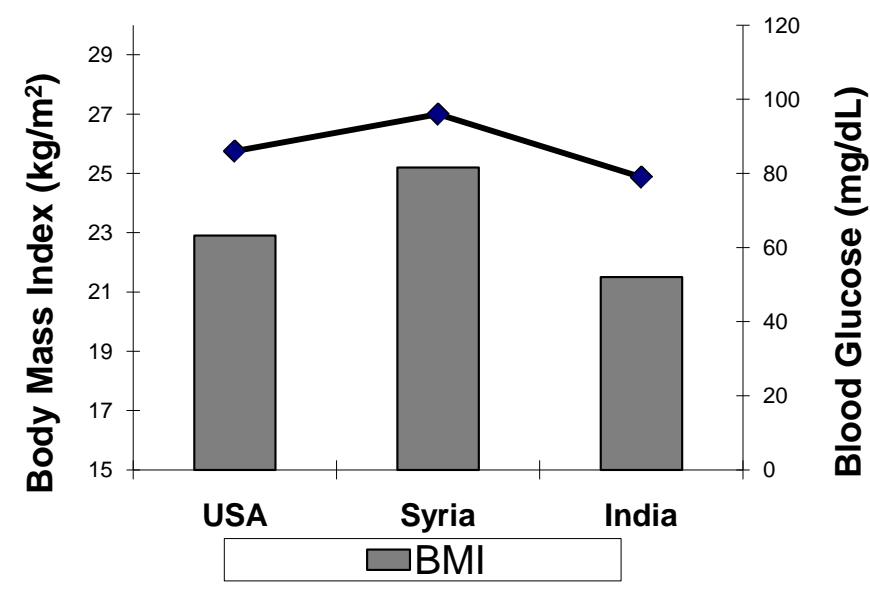

Fig. (1). Blood Glucose and BMI 
recently published INTERHEART study. The INTERHEART study evaluated potentially modifiable risk factors associated with myocardial infarction in 52 countries and confirmed the importance of these risk factors in the development of cardiovascular disease [13]. As would be expected, the number of young adults evaluated in the INTERHEART study was low (percent of patients under 40 years of age by geographic region ranged from $2.7 \%$ to $11.2 \%$ ). As previously mentioned, a unique aspect of this study is the fact that a young adult population was specifically targeted to look at cardiovascular risk factors.

One approach to affect the human and economic burden of cardiovascular disease for a nation is to reduce cardiovascular risk, especially among the young. Intervention programs that are either multifaceted or targeted may reduce disease burden. The results from our study suggest that a focused interventional program could be developed for each country. The advantage of such a program, especially, for countries with limited resources is that such a program may not be necessarily cost prohibitive. The disadvantage to a targeted approach is that not all cardiovascular risk factors are addressed.

If a targeted approach is taken to reduce CVD, then for a country like India, university student populations could be targeted to increase their overall amount of exercise. Besides the overall cardiovascular benefit of exercise, a secondary benefit would likely be an increase in HDL. One recommendation to increase HDL is to exercise [7]. Interestingly, in the INTERHEART trial the amount of exercise reported from South Asia was very similar to other geographic regions [13]. Our data may represent a unique population within South Asia that exercise less.

Perhaps the most important targeted approach for Syria is smoking. Clearly from our data, Syria had the highest number of smokers among the three sites. These findings are substantiated from data from WHO and the INTERHEART Study showing a high incidence of smoking in the Middle East. ${ }^{13}$ In addition, a recent report by the World Health Organization details smoking rates in adults by gender. In Syria, $62.0 \%$ of males and $21.0 \%$ of females smoke, whereas $32.7 \%$ of males and $1.4 \%$ of females smoke in India, and $22.3 \%$ of males and $17.4 \%$ of females in the U.S [14]. Of interest, a recent decree from the Syrian government will put into law a ban on smoking in many public places [15]. On another positive note, smoking is also very prevalent in South Asia, however we did not see a high prevalence in the university population we studied in India. If this finding is confirmed at other university settings this may bode well for the future for India.

Another concern for Syria is the high mean body mass index which is considered overweight and is likely the reason for the elevated glucose levels observed. As previously mentioned 39\% of subjects had impaired fasting glucose according to ADA definition. The reason for the elevated BMI is not known but likely relates in part to diet. Although the Mediterranean type diet typical in Syria is heart healthy it is also high in calories which may contribute to the observed elevated BMI. Another explanation may be the influence of "Western" style food. Clearly though, in addition to smoking another potential target area is weight loss, which should also have an added benefit of reducing glucose levels.

In regard to the United States, the significantly higher triglyceride levels as compared to the other countries could be a future target for intervention. The reasons for the higher triglyceride levels are unknown but may relate either to the higher consumption of alcohol or dietary aspects. Perhaps the high prevalence of fast food restaurants in the United States may contribute to this finding.

A unique aspect of this study is that university students were evaluated. Previous studies have looked at education level to determine if education provides a protective effect against cardiovascular disease. The most recent study based on the global Reduction of Ahterothrombosis for Continued Health (REACH) Registry suggest that attained education level was protective in high income countries but not in low and middle income countries [16]. Our results support these findings in that the country with the lowest income, Syria, appear to have the highest number of risk factors: smoking; BMI; glucose (including highest percent of subjects with impaired fasting glucose levels); and family history. Rapid urbanization, westernization, and targeted advertisements that may promote unhealthy life styles may play a role in these findings [16]. Obviously, based on our data and previous data cardiovascular risk reduction programs needs to include the educated population, especially in low income countries and among university students.

There are a number of limitations in regards to this study. The design of the study obviously allows for selection bias, as the subjects enrolled in the study were university students. In addition, it can be argued that the population we studied is the educational elite. Therefore, the applicability of these findings to other young populations outside this setting is not valid. Additional studies are planned to evaluate these populations including comparison to other university settings (for example, state funded, community colleges, etc.). Despite this limitation, these findings do give insight into a young university population. Another limitation of this study as previously mentioned was the point-of-care testing device used for cholesterol measurement was unable to quantify triglyceride $<50 \mathrm{mg} / \mathrm{dL}$. By default then, when triglyceride levels were $<50 \mathrm{mg} / \mathrm{dL}$, LDL levels were not calculated. Of the 296 subjects enrolled in the trial, $36 \%$ of subjects were reported to have TG $<50 \mathrm{mg} / \mathrm{dL}$ and this percentage was similar for all the countries (India 43\%, Syria 30\%, United States $32 \%, \mathrm{p}>0.05)$. We handled these findings by substituting the value of $49 \mathrm{mg} / \mathrm{dL}$ for the triglyceride level and then calculating the LDL. It is likely that by doing this we could have artificially inflated the triglyceride and LDL values, however, this introduced bias was similar across the three sites. In addition, the calculated LDL and triglyceride levels are considered low and does not suggest increase cardiovascular risk.

\section{CONCLUSION}

Cardiovascular disease is a significant cause of mortality worldwide. Without a significant intervention to reverse this disturbing trend, cardiovascular disease will likely reach epidemic proportions. It is extremely important to aggres- 
sively tackle cardiovascular disease in young adults, prior to the development of vascular complications. The results of this pilot study demonstrated that cardiovascular risk factors are apparent in a selected young educated population and the prevalence of risk factors may be different for different parts of the world. Our findings can be used to justify future research evaluating cardiovascular risk factors in various parts of the world among both the educated and non-educated young. Once further research is conducted, interventional programs may be established (customized to target the most prevalent risk factors for a particular country and perhaps socioeconomic and education status) which may help decrease the continuous rise in cardiovascular morbidity and mortality by reaching out to the young population.

\section{DISCLOSURES}

The authors of this study disclose no relevant financial relationships with commercial interests.

\section{ACKNOWLEDGEMENT}

The authors would like to thank the following pharmacy students that played a key role in data collection aspect of the study: Kalamoon University - Sawsan Abou Trab, Nour Kouli, Nour Lutfi, Nour Alkhateeb, and Rouba Ammar; Kakatiya University - Nagulu Malothu, Venkatesham Allenki, Uday Kiran Veldandi, Shankaraiah Puligilla, and Ramanarsimha Reddy Anreddy; University of Michigan - Yi Liu, Kendra Yum, Jenna Kast, Erin O’Neill, Hiep Ho, Yoon Chang, Tien Huynh, and Akinyemi Oni-Orisan.

\section{REFERENCES}

[1] World Health Organization. Cardiovascular Disease. Available at: http://www.who.int/mediacentre/factsheets/fs317/en/ Accessed June, 2010.
[2] Lopez AD, Mathers CD, Ezzati M, et al. Global burden of disease and risk factors. New York, NY: Oxford University Press 2006.

[3] World Health Organization. The World Health Report: Making a Difference. Geneva: World Health Organization, 1999.

[4] Murray CJL, Lopez AD. The Global Burden of Disease: A comprehensive assessment of mortality and disability from diseases, injuries and risk factors in 1990 and projected to 2020. Cambridge, MA: Harvard University Press 1996.

[5] Prabhakaran P, Ajay VS, Prabhakaran D, et al. Global cardiovascular disease research survey. J Am Coll Cardiol 2007; 50: 2322-8.

[6] http://www.cdc.gov/nchs/nhanes.htm - Accessed January 5, 2010

[7] Expert Panel on Detection, Evaluation, and Treatment of High Blood Cholesterol in Adults: Executive summary of the Third Report of the National Cholesterol Education Program (NCEP) Expert Panel on Detection, Evaluation, and Treatment of High Blood Cholesterol in Adults (Adult Treatment Panel III). JAMA 2001; 285: 2486-2497.

[8] National High Blood Pressure Education Program Coordinating Committee. The seventh report of the Joint National Committee on Prevention, Detection, Evaluation, and Treatment of High Blood Pressure: The JNC 7 report. JAMA 2003; 289: 2560-72.

[9] Standards of medical care in diabetes--2009. Diabetes Care 2009; 32 Suppl 1: S13.

[10] World Health Organization. http://apps.who.int/bmi/index.jsp?intro Page=intro_3.html. Accessed February 16, 2010.

[11] Misra A, Misra R, Wijesuriya M, Banerjee D. The metabolic syndrome in south asians: Continuing escalation possible solutions. Indian J Med Res 2007; 125: 345.

[12] Misra A, Khurana L. Obesity and the metabolic syndrome in developing countries. J Clin Endocrinol Metab 2008; 93: S9.

[13] Yusuf S, Hawken S, Ounpuu S, et al. Effect of potentially modificable risk factors associated with myocardial infarction in 52 countries (the INTERHEART study): case control. Lancet 2004; 364: 937-52.

[14] WHO Report on the Global Tobacco Epidemic, 2009. Implementing smoke-free environments. Accessed 3/5/2010: http://whqlibdoc. who.int/publications/2009/9789241563918_eng_full.pdf)

[15] www.sana.sy/print.html?sid=249130\&newlang=ara - Accessed January 19, 2010.

[16] Goyal A, Bhatt DL, Steg G, et al. Attained educational level and incident atherothrombotic events in low- and middle-income compared with high-income countries. Circulation 2010; 122: 1167-75. 